\title{
Characterization and determination of holin protein of Streptococcus suis bacteriophage SMP in heterologous host
}

\author{
Yibo Shi, Yaxian Yan*, Wenhui Ji, Bin Du, Xiangpeng Meng, Hengan Wang and Jianhe Sun*
}

\begin{abstract}
Background: Holins are a group of phage-encoded membrane proteins that control access of phage-encoded endolysins to the peptidoglycan, and thereby trigger the lysis process at a precise time point as the 'lysis clock'. SMP is an isolated and characterized Streptococcus suis lytic phage. The aims of this study were to determine the holin gene, HoISMP, in the genome of SMP, and characterized the function of holin, HoISMP, in phage infection.

Results: HolSMP was predicted to encode a small membrane protein with three hydrophobic transmembrane helices. During SMP infections, HoISMP was transcribed as a late gene and HolSMP accumulated harmlessly in the cell membrane before host cell lysis. Expression of HoISMP in Escherichia coli induced an increase in cytoplasmic membrane permeability, an inhibition of host cell growth and significant cell lysis in the presence of LySMP, the endolysin of phage SMP. HoISMP was prematurely triggered by the addition of energy poison to the medium. HolSMP complemented the defective $\lambda S$ allele in a non-suppressing Escherichia coli strain to produce phage plaques.
\end{abstract}

Conclusions: Our results suggest that HolSMP is the holin protein of phage SMP and a two-step lysis system exists in SMP.

Keywords: Streptococcus suis, Bacteriophage, Holin, Lysin

\section{Background}

Holin-lysin lysis systems typically exist in the doublestranded DNA bacteriophages for termination of their growth cycle and release of viral progeny through host cell lysis. By accumulating and forming lesions in the cytoplasmic membrane, holins control access of phageencoded endolysins to the peptidoglycan and thereby trigger lysis of the host cell at a precise time point. This process determines the length of the infection cycle and is known as the 'lysis clock'.

It is known that holins do not share sequence similarity, although they do have some common characteristics. Firstly, most holins are encoded by the gene adjacent to the endolysin gene. Secondly, at least one hydrophobic transmembrane domain (TMD) occurs in all holins.

\footnotetext{
* Correspondence: yanyaxian@sjtu.edu.cn; sunjhe@sjtu.edu.cn Department of Animal Science, School of Agriculture and Biology, Shanghai Jiao Tong University, Shanghai Key Laboratory of Veterinary Biotechnology, 800 Dongchuan Road, Shanghai, People's Republic of China
}

Thirdly, holins have a highly charged, hydrophilic, Cterminal domain. By identifying these characteristics, it is possible to predict putative holins. Holins can be grouped into three classes by topology. Class I holins, such as bacteriophage $\lambda \mathrm{S}$ protein [1] and Staphylococcus aureus phage P68 hol15 protein [2], generally have more than 95 residues and form three TMDs. Class II holins, such as the $\mathrm{S}$ protein from lambdoid phage 21 [3] and the Hol3626 protein from Clostridium perfringens bacteriophage $\Phi 3626$ [4], are smaller (65 to 95 residues) and form two TMDs. Class III holins, such as the holin of ФСР39O and ФCP26F [5], just have one TMD in the central region of the molecule. The schedulings of lysis time by some holin genes are specified by the dual-start model. In the dual-start model, the holin gene is an open reading frame that encodes two proteins, holin and antiholin, with opposing functions that are responsible for the accurate timing of the endolysin release [6,7]. For example, the prototype class I holin

\section{C) Biomed Central}

(C) 2012 Shi et al; licensee BioMed Central Ltd. This is an Open Access article distributed under the terms of the Creative Commons Attribution License (http://creativecommons.org/licenses/by/2.0), which permits unrestricted use, distribution, and reproduction in any medium, provided the original work is properly cited. 
gene, the $S$ gene of bacteriophage $\lambda$ encodes not only the effector holin, S105, but also an inhibitor, S107, with a $\mathrm{Met}_{1}-\mathrm{Lys}_{2}-\mathrm{Met}_{3} \ldots$ extension at the terminus. An sdi (site-directed initiation) structure near the 5 ' end of the $S$ gene controls translational initiations from the two initiator codons and determines the ratio of holin to antiholin.

Holins from bacteriophages infecting Gram-negative bacteria have been widely studied, especially bacteriophage $\lambda$ [8], bacteriophage T4 [9] and bacteriophage PRD $[10,11]$. In Gram-positive bacteria, several studies on holins have been conducted in phage infecting host cells such as Staphylococcus aureus [2,12], Lactococcus lactis [13,14], Lactobacillus fermentum [15], Streptococcus thermophilus [16], Streptococcus pneumoniae [17,18], Listeria monocytogenes [19] and Bacillus cereus [20]. However, no studies on holins from bacteriophages infecting Streptococcus suis (S. suis) have been reported

S. suis is an important pathogen of pigs causing arthritis, endocarditis, meningitis, pneumonia and septicemia [21]. Thirty-five serotypes (types 1 to 34 and 1/2) based on capsular antigens are currently known. Serotype 2 is considered the most virulent and prevalent type in diseased pigs in China. SMP, an S. suis serotype 2 lytic phage, was isolated and characterized in our previous work. Analysis of the complete genomic sequence (GenBank: EF116926) revealed the presence of a putative holin-lysin lysis system [22], thus providing further evidence that this is the universal mechanism to schedule host lysis for dsDNA phages. Phage-encoded lysins which could degrade peptidoglycan of Gram-positive bacteria exogenously have a bright future as potential therapeutic agents [23]. The extracellular lytic activities of LySMP, the putative endolysin of SMP, on S suis and its biofilm have been tested and confirmed [24,25], and HolSMP, the putative holin of SMP, was also showed synergistic antibacterial activity against $S$. suis with LySMP in our recent work (data not published). However, the exact structure and function of HolSMP remained to be investigated. The inability to isolate bacteria lysogenic for SMP limits the functional analysis of the holin gene. Fortunately, holin-dependent induction of membrane lesions is nonspecific, and this enables testing of holins encoded by bacteriophages infecting Gram-positive bacteria in Escherichia coli (E. coli) [26]. Therefore, in this study, the putative holin, HolSMP, was functionally analyzed in E. coli.

\section{Results and discussion}

\section{Computational predictions and analyses of HolSMP}

The complete 36,126-bp sequence of phage SMP has 48 open reading frames (ORF). ORF42, designated LySMP, encodes the putative endolysin. ORF43, which is $429 \mathrm{bp}$ long and upstream of the lySMP gene, is predicted to encode a putative holin protein, designated HolSMP (Figure 1A). There is a 96-bp sequence located between HolSMP and LySMP. A ribosome-binding site (RBS) was identified upstream of the first start codon (Figure 1B). HolSMP consists of 142 amino acids (15.7 kDa) and shows $88 \%$ similarity to the sequence of a putative holin of Streptococcus phage MM1 (accession number NP 150180), and exhibits the characteristics of phageholin_4 superfamily. The results of PredictProtein, TmHMM and SOSUI analysis suggested that HolSMP is a membrane protein with traits typical of a holin. HolSMP has three putative hydrophobic TMDs and its $\mathrm{N}$-terminus extends into the periplasm (Figure $1 \mathrm{C}$ ). HolSMP is rich in methionine residues. Five of them, Met $_{1}$, Met $_{3}$, Met $_{4}$, Met $_{8}$ and Met ${ }_{15}$, are located upstream of $\mathrm{TMD}_{1}$. Met $t_{40}$ is within $\mathrm{TMD}_{1}$. Met ${ }_{53}$ is located between $\mathrm{TMD}_{1}$ and $\mathrm{TMD}_{2}$, and the three other Met codons, $\mathrm{Met}_{73}, \mathrm{Met}_{74}$ and $\mathrm{Met}_{85}$, are all within $\mathrm{TMD}_{2}$ (Figure 1C). HolSMP has a hydrophilic C-terminus with several charged amino acids, whereas the $\mathrm{N}$-terminus has three positively charged amino acids and only one negatively charged residue. HolSMP shares structural characteristics of holins, and HolSMP should be assigned to class I.

The dual-start model is still to be explored in holins of SMP although it is found in most other bacteriophages. Although HolSMP is rich in Met residues at the $\mathrm{C}$-terminus, the Lys residue is absent at the $\mathrm{N}$-terminus $\left(\mathrm{Met}_{1}-\mathrm{Val}_{2}-\mathrm{Met}_{3}-\mathrm{Met}_{4} \ldots\right)$, and no sdi structure has been found near the 5' end of HolSMP. The western blot results of HolSMP expression in both homologous and heterologous hosts, showed a single detectable band at the expected position (Figures $2 \mathrm{~B}$ and $3 \mathrm{~B}$ ), but whether HolSMP is the unique product of HolSMP should be investigated further by methods such as the toeprinting assay. The present data do not support the dual-start model for phage SMP. In bacteriophge $\lambda$ S105 protein, a unique but crucial cysteine is shown to cause the formation of disulfide-linked dimmers under oxidative conditions, which suggested a model of holin to form lesion in the membrane [27], but no cysteine is occupied by HolSMP, which might imply a different lesion-formation model of holin from the one of S105 protein.

\section{Expression of HolSMP in S.suis during phage SMP infection}

The one-step growth curve of SMP has been studied previously and showed a latent period of at least 20 min and a rise period of $120 \mathrm{~min}$ [22]. To determine the transcription of HolSMP in SMP-infected S. suis, samples were collected at $0,5,10,15,20,40,60,80$, 100 and $120 \mathrm{~min}$ after infection. Total RNA was extracted from the samples and was reverse transcribed into cDNA, and fragments of HolSMP (473 bp) 

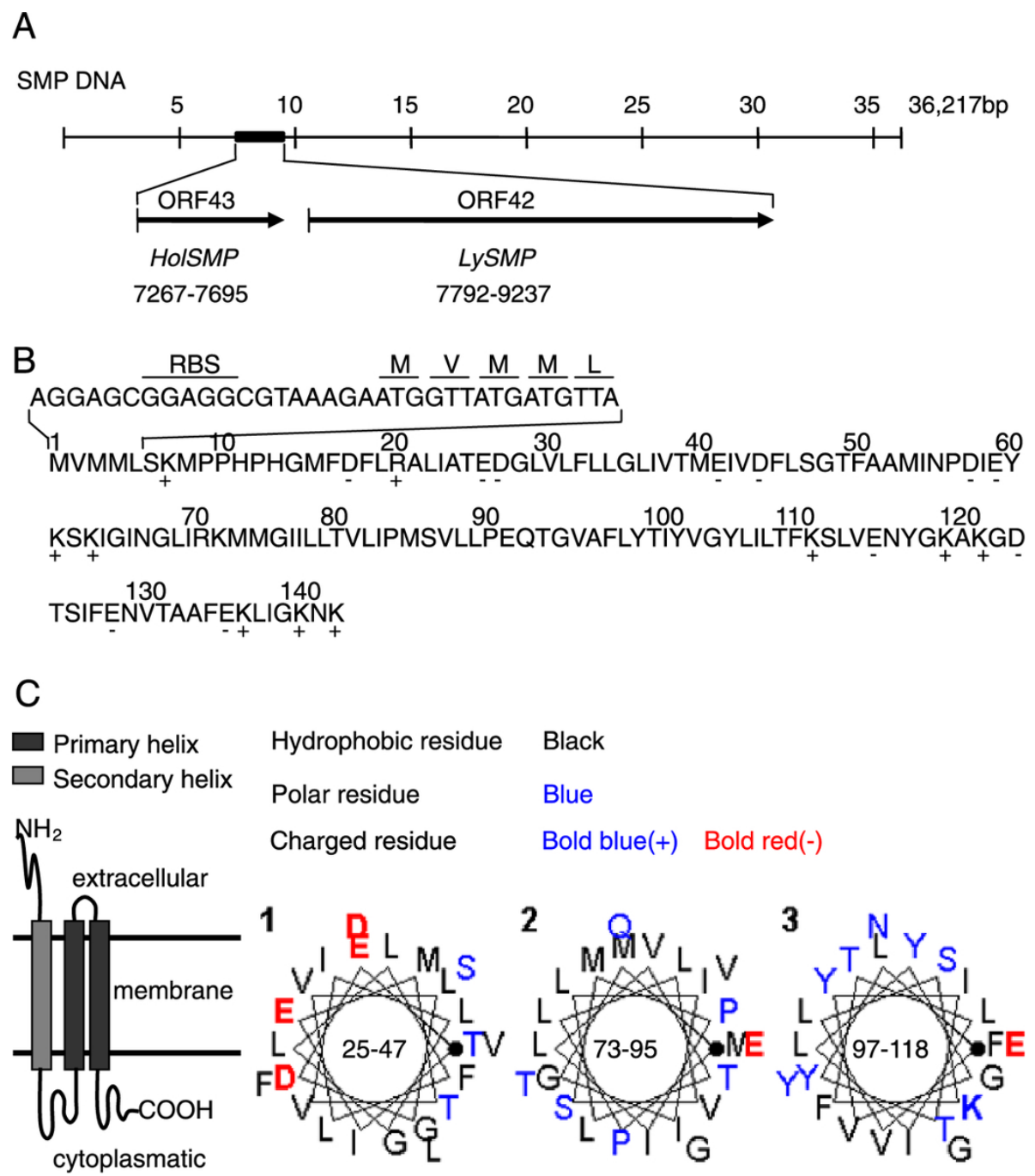

Figure 1 ORF43 from the complete sequence of SMP was analyzed. (A) Location of LySMP (ORF42) and Ho/SMP (ORF43), which are likely to comprise the putative holin-lysin lysis system of SMP. (B) The primary sequence of the HolSMP product. Charged residues are indicated below the sequence. (C) Secondary structure of HolSMP predicted by SOSUI.

and glyceraldehydes-3-phosphate dehydrogenase (GAPDH, $223 \mathrm{bp}$ ) gene were amplified by PCR. The electrophoresis result showed that the GAPDH gene of host cells could be detected in all samples, while the HolSMP gene could only be detected after $20 \mathrm{~min}$. Moreover, the transcript level of HolSMP kept rising rapidly before $60 \mathrm{~min}$ (Figure $2 \mathrm{~A}$ ) and then started to decline. Real-time quantitative PCR relatively quantified the accumulation of HolSMP mRNA (Table 1). The results showed that HolSMP transcripts were undetectable before $15 \mathrm{~min}$. A sharp increase appeared before $60 \mathrm{~min}$. The numbers of HolSMP transcripts at 20,40 and $60 \mathrm{~min}$ were 10,523 and 1,722 times greater than at $15 \mathrm{~min}$, respectively. Decreased HolSMP transcript levels were observed after $60 \mathrm{~min}$ and lasted to the end of the experiment at $120 \mathrm{~min}$.
Further, to determine the product of HolSMP, host cells were collected at 20,40, 60, 80 and 100 min after infection and separated from cultures by centrifugation. It was notable that samples collected at 60 and $80 \mathrm{~min}$ were viscous, indicating the action of a holinlysin lysis system and the release of progeny phages. The membrane fraction was extracted from each sample and examined by western blotting. One single band corresponding to HolSMP (15.7 kDa) appeared at the expected position of each lane except for the sample collected at $20 \mathrm{~min}$ (Figure 2B), suggesting that HolSMP is located in the membrane of phage-infected host cells. Accumulation of HolSMP in the membrane was found before $60 \mathrm{~min}$. With a reduction of HolSMP transcripts and destruction of host cells, the amount of HolSMP in the membrane also started to reduce. All 


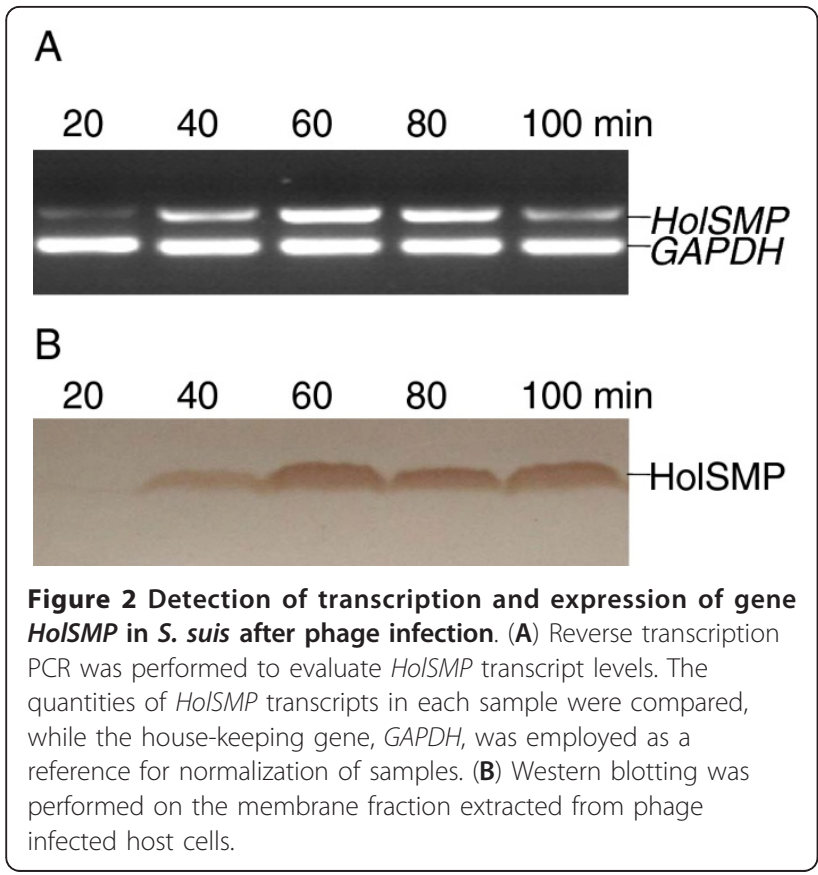

of the above results show that HolSMP is a late gene. Transcription and expression of HolSMP does not occur during the early stage of infection but is highly up-regulated when progeny phages are released.

\section{Expression of HolSMP in E. coli}

E. coli is a convenient host for the investigation of holin proteins from phage that infect Gram-positive bacteria [26]. Therefore, the functional identification of putative holin protein HolSMP was performed in BL21(DE3) pLysS (pEXH1) strains. The plasmid pEXH1, containing the HolSMP gene sequence, was constructed. BL21 (DE3)pLysS (pEXH1) was created and growth of transformants was monitored after induction with $\beta$-D-thiogalactopyranoside (IPTG) (Figure 3A). Growth inhibition of induced cells occurred from 25 to $40 \mathrm{~min}$ and was followed by cell lysis with the $\mathrm{OD}_{600}$ value reducing from 0.85 to 0.24 . Toxicity of HolSMP to BL21 (DE3)pLysS cells was further proven by the viability assay of induced cells. It was showed that the number of viable cells began to decrease at $10 \mathrm{~min}$ and a three-logunit drop was observed (Figure 3A). Moreover, as is characteristic of all holins, HolSMP could be triggered prematurely by the addition of energy poison, potassium cyanide (KCN, $10 \mathrm{mM}$ ) (Figure 3A). To determine the kinetics of HolSMP expression, total cellular protein samples were prepared every $8 \mathrm{~min}$, and the accumulation of HolSMP was determined by western blotting. The results showed that a $16 \mathrm{kDa}$ species, consistent with the predicted mass of HolSMP, was detected in samples taken after $24 \mathrm{~min}$, but not before (Figure 3B).

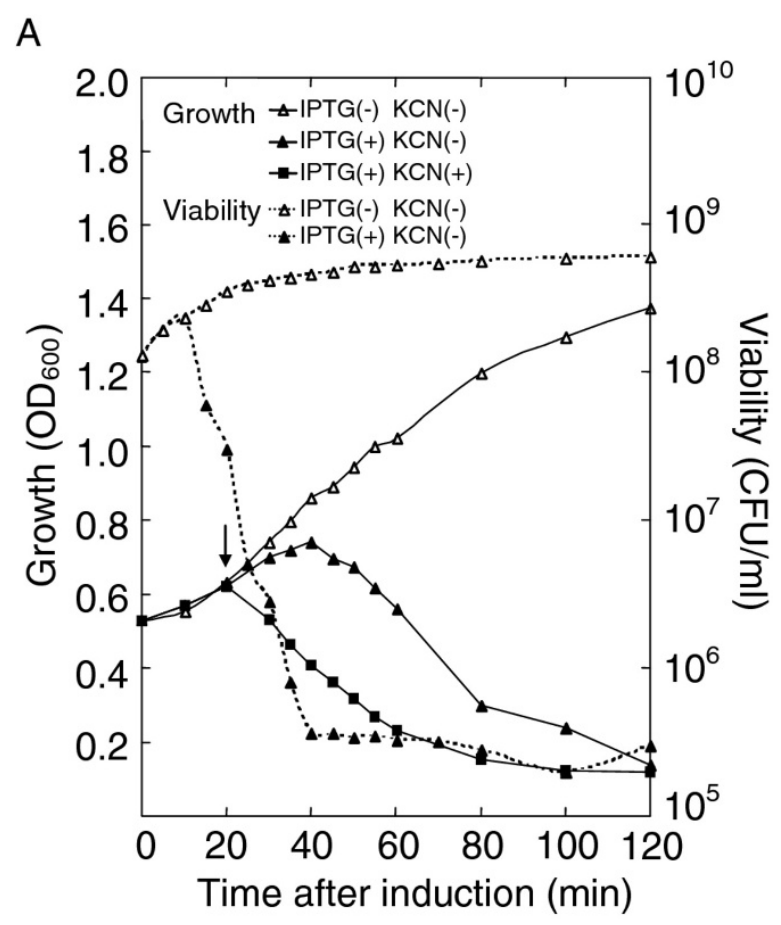

B

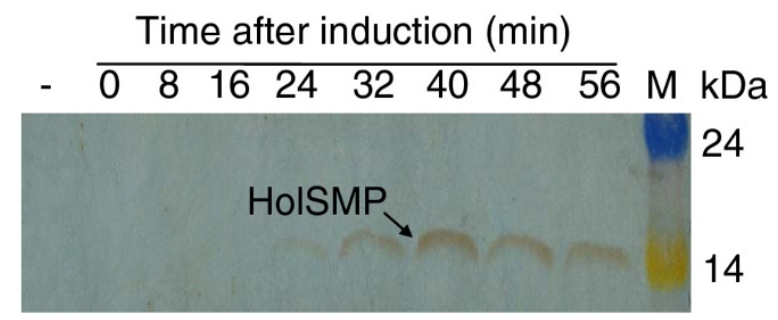

Figure 3 HolSMP is toxic to $E$. coli and cyanide triggers the lethal potential of HolSMP. (A) The growth and viability assays of BL21(DE3)pLysS harboring plasmid pEXH1 were performed after addition of IPTG. At the time indicated by the arrow, KCN was added to the culture. Non-induced BL21(DE3)pLysS (pEXH1) was also monitored as control. (B) Western blotting was performed on total cellular protein of IPTG induced BL21(DE3)pLysS (pEXH1) to detect expression of HolSMP. Cultures of BL21(DE3)pLysS (pEX) were collected at $56 \mathrm{~min}$ after induction as a negative control (-). A size marker is shown in the last lane.

The level of HolSMP protein increased from 24 min to $40 \mathrm{~min}$ and then declined.

HolSMP accumulated in and damaged the membrane of expressing cells. In order to confirm the subcellular location of HolSMP in cells, cultures of BL21(DE3) pLysS (pEXH1) were collected 40 min after induction to isolate total cellular protein, cytoplasmic protein and membrane protein samples. As the negative control, protein fractions were also prepared from BL21(DE3) pLysS (pEX). Western blots revealed that the dark brown bands indicating HolSMP protein appeared in 
Table 1 Relative quantitation of HolSMP using the comparative $\mathrm{C}_{\mathrm{T}}$ method

\begin{tabular}{|c|c|c|c|c|c|}
\hline Time (min) & HolSMP Average $C_{T}$ & GAPDH Average $C_{T}$ & $\Delta \mathrm{C}_{\mathrm{T}} H$ HolSMP- GAPDH & $\Delta \Delta \mathrm{C}_{\mathrm{T}} \Delta \mathrm{C}_{\mathrm{T}}-\Delta \mathrm{C}_{\mathrm{T}, 0 \text { min }}{ }^{\mathrm{b}}$ & HolSMP Rel. to $0 \mathrm{~min}^{\mathrm{c}}$ \\
\hline 0 & $30.61 \pm 0.09$ & $16.64 \pm 0.03$ & $13.97 \pm 0.09$ & $0.00 \pm 0.09$ & $1.0(0.9-1.1)$ \\
\hline 5 & $30.66 \pm 0.03$ & $16.83 \pm 0.03$ & $13.83 \pm 0.04$ & $-0.14 \pm 0.04$ & $1.1(1.07-1.13)$ \\
\hline 10 & $29.60 \pm 0.05$ & $18.40 \pm 0.03$ & $11.20 \pm 0.06$ & $-2.77 \pm 0.06$ & $6.8(6.5-7.1)$ \\
\hline 15 & $24.54 \pm 0.03$ & $16.30 \pm 0.05$ & $8.24 \pm 0.06$ & $-5.73 \pm 0.06$ & $53.1(50.9-55.3)$ \\
\hline 20 & $21.27 \pm 0.02$ & $16.38 \pm 0.04$ & $4.89 \pm 0.04$ & $-9.08 \pm 0.04$ & $541.2(526.4-556.4)$ \\
\hline 40 & $17.69 \pm 0.03$ & $18.49 \pm 0.03$ & $-0.80 \pm 0.04$ & $-14.77 \pm 0.04$ & 27939.1 (27175.1-28724.6) \\
\hline 60 & $16.10 \pm 0.02$ & $18.61 \pm 0.05$ & $-2.51 \pm 0.05$ & $-16.48 \pm 0.05$ & 91405.9 (88292.3-94629.3) \\
\hline 80 & $16.42 \pm 0.03$ & $18.55 \pm 0.02$ & $-2.12 \pm 0.04$ & $-16.09 \pm 0.04$ & 69754.6 (67847.1-71715.6) \\
\hline 100 & $15.26 \pm 0.07$ & $16.56 \pm 0.04$ & $-1.30 \pm 0.08$ & $-15.27 \pm 0.08$ & 39511.9 (37380.6-41764.8) \\
\hline 120 & $17.94 \pm 0.03$ & $18.68 \pm 0.01$ & $-0.74 \pm 0.03$ & $-14.71 \pm 0.03$ & $26801.0(26249.5-27364.2)$ \\
\hline
\end{tabular}

a. The $\triangle C_{T}$ value is determined by subtracting the average GAPDH $C_{T}$ value from the average HolSMP $C_{T}$ value. The standard deviation of the difference is calculated from the standard deviation of the HoISMP and GAPDH values.

b. The calculation of $\Delta \Delta C_{\mathrm{T}}$ involves subtraction by the $\Delta \mathrm{C}_{\mathrm{T}}$ calibrator value. This is subtraction of an arbitrary constant, so the standard deviation of $\Delta \Delta \mathrm{C}_{\mathrm{T}}$ is the same as the standard deviation of the $\Delta C_{T}$ value.

c. The range given for HolSMP relative to 0 min is determined by evaluation the expression: $2^{-\Delta \Delta C T}$ with $\Delta \Delta C_{T}+s$ and $\Delta \Delta C_{T}-s$, where $s=$ the standard deviation of the $\Delta \Delta C_{T}$ value.

total cellular protein preparations and the membrane fraction of BL21(DE3)pLysS (pEXH1), but not in the cytoplasmic fraction or in the HolSMP-negative subcellular samples. This suggested that HolSMP accumulates in the membrane of E. coli, consistent with S. suis.

Changes in cell morphology during HolSMP expression after induction were explored by observing cells harboring plasmid pEXH1 with phase contrast microscopy. BL21(DE3) cells induced with IPTG appeared translucent and non-refractile, with a normal shape. The expected changes in transparency and refraction of cells were also observed in the cell wall after induction of the holin genes of phage $\lambda, \Phi 29$ and pneumococcal bacteriophage EJ-1 [17]. It has been reported that holin, even in the absence of lysin, is lethal for the host cell since it causes increased cytoplasmic membrane permeability, collapse of the membrane potential, inhibition of respiration and defective active transport. BL21(DE3) pLysS (pEXH1) cells also appeared translucent and nonrefractile after induction. Furthermore, BL21(DE3)pLysS $(\mathrm{pEXH1})$ cells were round and somewhat larger than normal cells. Therefore, ultrathin sections of induced BL21(DE3)pLys (pEXH1) cells were prepared and examined by electron microscopy. A subtle separation of the cytoplasmic membrane from the cell wall was observed in induced cells when the cells were still of normal size (Figure 4A and 4B). A dramatic expansion of cells and an indistinct cell wall could be observed about $1 \mathrm{~h}$ after induction (Figure 4C). The phenomenon was consistent with the phase contrast microscopy. BL21(DE3)pLysS harbors the pLysS plasmid, which carries the gene encoding T7 lysozyme. Therefore, it was deduced that the expression of HolSMP in BL21(DE3) pLys $\mathrm{S}$ compromised the cytoplasmic membrane, leading to the release of T7 lysozyme and further damage to the cell wall, resulting in the observed changes in shape and size. Our observations are indirect evidence that accumulation of HolSMP leads to lesion formation in the cytoplasmic membrane, through which some proteins might pass and execute their function.

\section{Determination of HoISMP as a holin protein}

LySMP, the SMP endolysin encoded by gene LySMP, cannot cause cell lysis without the assistance of holin. To further identify the physiological role of HolSMP as a holin protein for the release of endolysin, we coexpressed HolSMP with LySMP in BL21(DE3). The growth of clones co-transformed with pACEXL and pEXH1 were monitored after induction with IPTG (Figure 5). The results showed that cells co-transformed with pEX and pACEXL, expressing only LySMP, did not cause any host cells lysis. Co-transformation with pEXH1 and pACEX, expressing HolSMP, started to inhibit the growth of host cells as early as $10 \mathrm{~min}$ followed by a slow decrease in absorbance 20 min after induction. However, the coexpression of HolSMP and LySMP resulted in an abrupt decrease in absorbance from $20 \mathrm{~min}$. These results suggested that HolSMP does not significantly lyse cells, but might permit the release of LySMP, which results in the abrupt decrease in absorbance.

HolSMP complements an S-negative lysis-defective $\lambda$ phage mutant. It is known that holins are essential for endolysin $\mathrm{R}$ of the $\lambda$ phage to display lytic activity. Phage $\lambda$ cI857 $\operatorname{Sam} 7$ carries an amber mutation in the $S$ gene and, consequently, cannot trigger lysis of the infected host cells unless a suppressing $E$. coli strain is used. To further document the role of HolSMP, we carried out complementation tests using the non-suppressing strain BL21(DE3)pLysS (pEXH1) and phage $\lambda$ cI857 


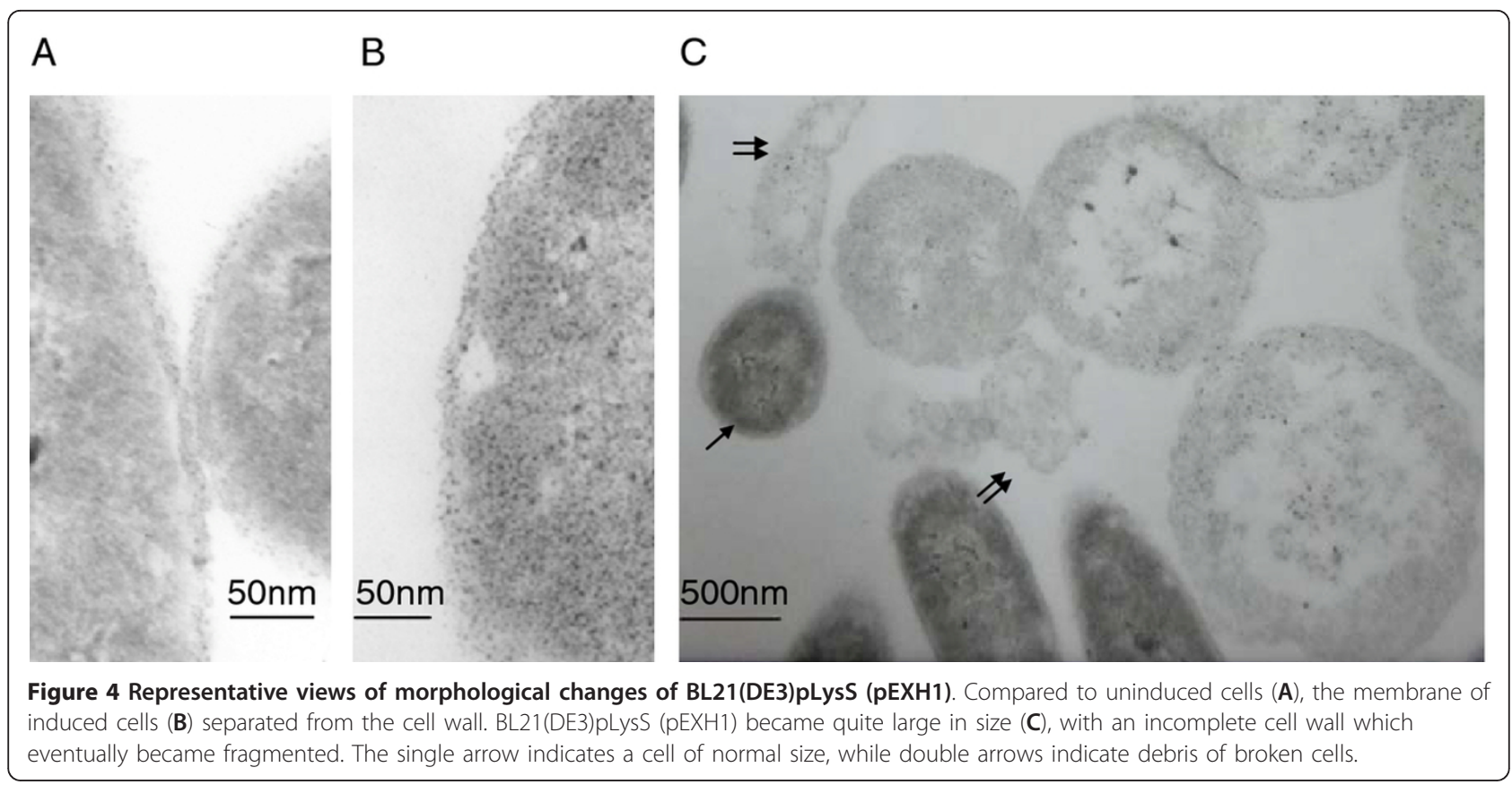

Sam7. BL21(DE3)pLysS (pEXH1), infected by phage $\lambda$ cI857 Sam7, was added to soft agar containing IPTG. Because the high efficiency of expression of the pET system may lead to hyper-expression of HolSMP and cell toxicity, the IPTG concentration was reduced to 0.1 $\mathrm{mM}$. This induces expression of HolSMP at sublethal levels. Plaques were observed when HolSMP was induced. No plaques formed on the plates of BL21(DE3)

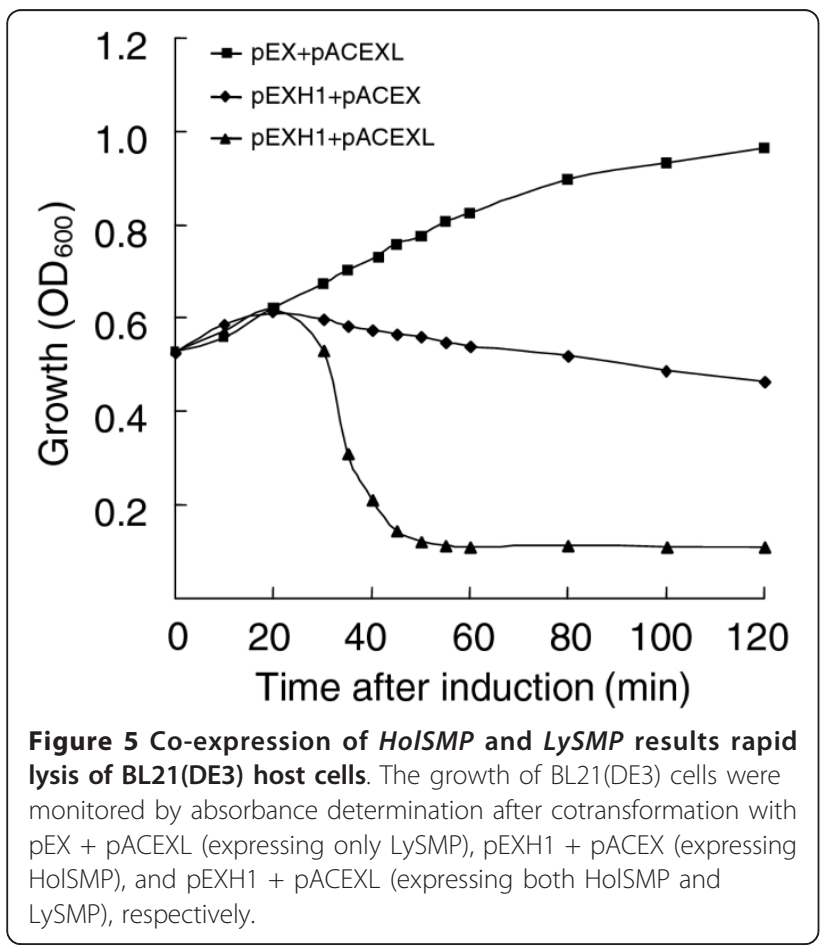

pLysS harboring plasmid pET-32a(+) used as a control plasmid for pEXH1. The defective bacteriophage $\lambda$ cI857 Sam7 can form plaques when grown on the suppressing strain VCS257. Moreover, plaques formed by phage $\lambda$ cI857 Sam7 on the complementation plates were larger and clearer than the supF host VCS257 plates (Figure 6 ). The result of the complementation test indicated that HolSMP is able to function as a holin protein and complement an S-negative lysis-defective $\lambda$ phage mutant.

\section{Conclusions}

From these results, protein HolSMP has been identified for the first holin of S. suis bacteriophage. The holin gene, HolSMP, located upstream of the endolysin gene is transcribed, expressed in S. suis after infection by phage SMP. HolSMP, a putative member of class I holins, accumulates on membrane of S. suis. At present, an S. suis strain lysogenic for SMP has not been isolated, and it is not easy to obtain HolSMP-defective SMP mutants. However, biological evidence for the holin-like character of HolSMP was obtained in a Gram-negative background. The HolSMP product caused cellular death, and changes in cell morphology could be accounted for by lesions in the membrane. By forming lesions in the cytoplasmic membrane, HolSMP permitted T7 lysozyme in BL21(DE3)pLysS, LySMP in BL21(DE3), and R in phage $\lambda$-infected cells to escape through the membrane and damage the cell wall. HolSMP shows the same host non-specificity and membrane energy sensitivity as other holins $[17,18]$. Thereby, the holin-lysin lysis system of SMP was also determined 


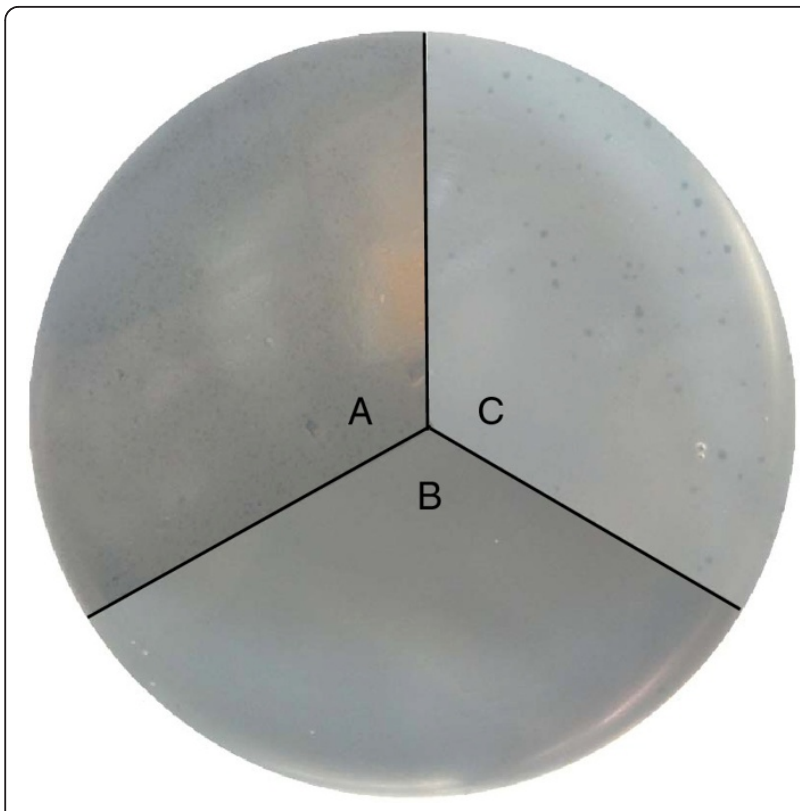

Figure 6 HoISMP functions as a holin to complement Snegative phage $\lambda$ cl857 Sam7. (A) Host cell lysis by phage $\lambda$ c1857 Sam7 requires a permissive host, featuring supF, such as VCS257. (B) Plaques will not be produced on plates with nonermissive host BL2 (DE3)pLysS. (C) With the expression of sub-lethal levels of HolSMP, large and clear plaques were observed on plate with host BL2(DE3) pLysS (pEXH1).

in this study. The HolSMP is able to trigger activity of the LySMP and release viral progeny through host cell lysis. Our further biochemical investigations will shed light of the mechanism of HolSMP action and the application of HolSMP in biopharmacy

\section{Methods}

Bacterial strains, phages, plasmids and growth conditions Bacterial strains, phages, and plasmids used in this study are listed in Table 2. S. suis SS2-H was grown in ToddHewitt broth (THB) or agar medium, supplemented with $2 \%(\mathrm{v} / \mathrm{v})$ newborn bovine serum, at $37^{\circ} \mathrm{C}$. Preparation of bacteriophage SMP was carried out as described previously [24]. Briefly, SMP was propagated on SS2-H by the double-layer agar plate method and eluted with phage buffer containing $100 \mathrm{mM} \mathrm{NaCl}, 8 \mathrm{mM} \mathrm{MgSO}_{4}$, $50 \mathrm{mM}$ Tris, $\mathrm{pH} 7.5$ and $0.1 \%(\mathrm{w} / \mathrm{v})$ gelatin. SMP was separated from the host by filtration. E. coli strains were cultured in standard Luria-Bertani medium (LB) supplemented with ampicillin $(100 \mu \mathrm{g} / \mathrm{ml})$ (LB-Ap) or chloramphenicol $(30 \mu \mathrm{g} / \mathrm{ml})(\mathrm{LB}-\mathrm{Cm})$ or both (LB-Ap-Cm), as appropriate. In BL21(DE3)pLysS, pLysS-encoded T7 lysozyme inhibits T7 RNA polymerase to reduce basal expression of target gene and is also able to degrade the peptidoglycan layer of the cell wall when membrane lesions exist. The suppressing strain VCS257 was cultured in LB supplemented with $10 \mathrm{mM} \mathrm{MgSO}_{4}$ and
$0.2 \%(\mathrm{w} / \mathrm{v})$ maltose for infection by phage $\lambda$ cI857 Sam7. Liquid cultures were incubated overnight at $37^{\circ} \mathrm{C}$ with shaking at $200 \mathrm{rpm}$.

\section{Computational analyses}

DNA and protein sequence homology alignments were performed using BLAST tools on NCBI. TMHMM http://www.cbs.dtu.dk/services/TMHMM/, SOSUI http://bp.nuap.nagoya-u.ac.jp/sosui/ and PredictProtein servers http://www.predictprotein.org were used to predict the transmembrane helices in HolSMP.

\section{DNA manipulation and plasmid construction}

DNA was isolated from SMP as described for phage $\lambda$ [28]. The HolSMP and LySMP genes were amplified by PCR from purified phage genomic DNA using primers designed listed in Table 3 . The amplified genes were then cloned into prokaryotic expression vectors using two restriction sites incorporated in the PCR primers. The ligation product was transformed into competent $E$. coli DH5 $\alpha$. Recombinant plasmids were extracted from transformants and sequenced. Plasmids were retransformed into competent E. coli BL21(DE3) or BL21(DE3) pLysS under selection. It should be noted that there are no tags coding sequences in the recombinant plasmids for which original physiological functions of HolSMP and LySMP remained

To construct plasmid pEXH1, HolSMP was amplified with oligonucleotides EXH1 and EXH3 to generate a PCR fragment (HolSMP-1) containing the RBS of plasmid pET-32a $(+)$. PCR using fragment HolSMP-1 as the template was performed to generate a final $495 \mathrm{bp} \mathrm{PCR}$ fragment (HolSMP-2) with primer EXH2 and the common reverse primer EXH3. HolSMP-2 was cloned into the $\mathrm{XbaI}$ and $\mathrm{BamHI}$ sites of pET-32a(+) to give plasmid pEXH1. Plasmid pEXL, harboring the LySMP gene, was constructed in the same as was plasmid pEXH1, using primer pairs EXH5/EXH4 and EXH2/EXH4.

The negative control plasmid pEX was constructed using the same strategy as for pEXH1, using primer SYB34 to introduce a stop codon at the beginning of HolSMP. The expression vectors PACEXL and PACEX were constructed by subcloning the HindIII-SphI fragment of pEXL (containing the T7 promoter, RBS, Lac operon and lysine gene) or pEX (containing T7 promoter, RBS, Lac operon and MCS) into HindIII-SphI digested pACYC184.

\section{RNA extraction from SMP-infected host cells}

To identify the transcription of the HolSMP gene in SMP-infected S. suis, exponentially growing SS2-H cells (about $\left.10^{9}\right)$ were infected with phage SMP $\left(10^{9}\right.$ $10^{10}$ plaque forming units $/ \mathrm{ml}$ ) at a multiplicity of infection of at least 10 . A sample, containing $10^{8}$ host 
Table 2 Bacterial strains, phages and plasmids used in this study

\begin{tabular}{|c|c|c|}
\hline $\begin{array}{l}\text { Strains, phages or } \\
\text { plasmids }\end{array}$ & Genotype and relevant features & Source \\
\hline Strains $\mathrm{DH} 5 \alpha$ & 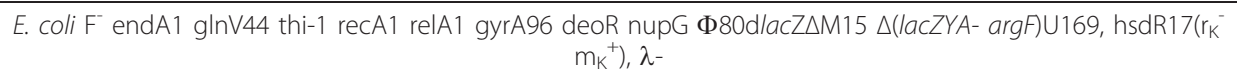 & Tiangen \\
\hline BL21(DE3) & E. coli FompT hsdS $\left(r_{B}^{-} m_{B}^{-}\right)$gal dcm (DE3) & Tiangen \\
\hline BL21(DE3) plysS & E. coli FompT hsdS $\left(r_{B}^{-} m_{B}^{-}\right)$gal dcm (DE3) pLysS $\left(\mathrm{Cm}^{\mathrm{R}}\right)$ & Tiangen \\
\hline VCS 257 & DP50 supF[supE44 supF58 hsd53( $\left.\mathrm{r}_{\mathrm{B}} \mathrm{m}_{\mathrm{B}}\right)$ dap D8lacY1 glnV44 $\triangle$ (gal-uvrB)47 tyrT58 gyrA29 tonA53 $\Delta$ (thyA57)] & Stratagene \\
\hline SS2-H Phages & Streptococcus suis, serotype 2 & Lab stock \\
\hline SMP & Wild type phage of S. suis & Lab stock \\
\hline$\lambda$ cl857 Sam7 & clts857, Sam7,Lac promoter for expression of cloned genes, phage of E. coli & Stratagene \\
\hline \multicolumn{3}{|l|}{ Plasmids } \\
\hline pET-32a(+) & $\begin{array}{c}\text { Expression vector containing hybrid T7-lacPO promoter, } \Phi 10 \text { ribosome binding site, and lacl gene (pBR322 } \\
\text { derivative), ampicillin resistance }\end{array}$ & Novagen \\
\hline pACYC184 & Low copynumber cloning vector; p15A ori; $\mathrm{Tc}^{+} ; \mathrm{Cm}^{+}$ & Lab stock \\
\hline pEXH1 & Derivative of pET-32a(+); whole ORF of HolSMP gene, HolSMP(429), inserted behind RBS of pET-32a(+) & This study \\
\hline $\mathrm{pEXL}$ & Derivative of pET-32a(+); LySMP fragment inserted behind RBS of pET-32a(+) & This study \\
\hline pEX & Derivative of pET-32a(+); pEXH1 ( $\triangle$ HolSMP) & This study \\
\hline PACEXL & Hind III-Sphl fragment of pEXL subcloned into Hind III-Sphl sites of pACYC184 & This study \\
\hline PACEX & Hind III-Sphl fragment of pEX subcloned into Hind III-Sphl sites of pACYC184 & This study \\
\hline
\end{tabular}

cells, was collected prior to addition of phage and immediately centrifuged at $13,000 \times g$ for $1 \mathrm{~min}$ to pellet cells. The rest of the reaction was incubated at $37^{\circ} \mathrm{C}$ for $15 \mathrm{~min}$ and centrifuged at $13,000 \times g$ for $1 \mathrm{~min}$. The cell pellet was re-suspended gently with THB and incubated at $37^{\circ} \mathrm{C}$ with shaking at $150 \mathrm{rpm}$. Samples, containing $10^{8}$ cells, were collected as described above at $5,10,15,20,40,60,80,100$ and 120 min after infection. Cell pellets were snap frozen in liquid nitrogen as soon as the supernatant was discarded, and stored at $-20^{\circ} \mathrm{C}$ until RNA extraction. Total RNA of all samples was extracted simultaneously with the RNA extraction kit (Omega). Contaminating DNA was removed by digestion. Downstream cDNA synthesis was performed when DNA from SMP and host cells could not be detected by PCR.

Table 3 Primers used in this study ${ }^{a}$

\begin{tabular}{cc}
\hline Primers & Sequences \\
\hline EXH1 & AACTTAAGAAGGAGATATACATatggttatgatgtta \\
EXH2 & ACGCTCTAGAAATAATTTGTTAACTTAAGAAGGAG \\
EXH3 & CGCGGATCCctttgattagtttcatttat \\
EXH4 & CGCGGATCCttatcttaaactggtttt \\
EXH5 & TTAACTTAAGAAGGAGGAAATCatgacaatcaacatt \\
SYB34 & AACTTAAGAAGGAGATATACATatggttatgtgatta \\
SYB35 & ttccgatgtcagtgctt \\
SYB36 & ttggctttgccgtagtt \\
GAPDH1 & actatcggcttggtaatcccagat \\
GAPDH2 & atgaaccgaatgagatacctacga \\
\hline
\end{tabular}

a. New restriction sites and RBS are indicated by underline and shadow, respectively. Lowercase letters represent sequences corresponding to that of HolSMP.

\section{Reverse transcription PCR}

The transcript levels of HolSMP were determined visually by reverse transcription PCR. MMLV reverse transcriptase (25 $\mathrm{U}$ ) and random primers (TakaRa) were used for cDNA synthesis. The $S$. suis housekeeping gene $G A P D H$ was employed as a reference for normalization of samples.

Two pairs of primers, EXH1/EXH3 and GAPDH1/ GAPDH2 (Table 3), were used to amplify HolSMP and $G A P D H$, respectively. PCR was carried out in a final volume of $25 \mu \mathrm{l}$, containing $2 \mu \mathrm{l}$ cDNA (1:5 dilution), $0.4 \mu \mathrm{M}$ of each primer, and $12.5 \mu \mathrm{l} 2 \times$ PCR mix (Dongsheng Biotech). Amplification was performed for 28 cycles with annealing temperatures of $57.5^{\circ} \mathrm{C}$ and $60^{\circ} \mathrm{C}$ for HolSMP and GAPDH, respectively. The volume of each HolSMP PCR product loaded for electrophoresis was adjusted based on the corresponding GAPDH fragments (223 bp). Gels were visualized with an image analysis system after electrophoresis. Then, bands corresponding to the HolSMP gene (473 bp) in each lane were compared.

\section{Real-time quantitative PCR}

Further relative quantification of transcript levels of HolSMP was performed by real-time quantitative PCR. Primers SYB35 and SYB36 were designed using primer 5.0 software to amplify 116 bp of HolSMP (Table 3). The $S$. suis housekeeping gene GAPDH was employed as a reference for normalization of samples. PCR was carried out with a PTC-200 PCR instrument (Bio-Red, Hercules, CA) and MJ option Monitor analysis system. PCR was carried out in a final volume of $50 \mu \mathrm{l}$, containing 2 $\mu \mathrm{l}$ cDNA (1:5 dilution), $0.4 \mu \mathrm{M}$ of each primer, and $1 \times$ 
SYBR premix EX taq II (Takara). Amplification was performed over 40 cycles of $5 \mathrm{~s}$ at $95^{\circ} \mathrm{C}, 30 \mathrm{~s}$ at the annealing temperature $\left(57.5^{\circ} \mathrm{C}\right.$ for $\operatorname{HolSMP}$ and $60^{\circ} \mathrm{C}$ for $G A P D H)$, and $10 \mathrm{~s}$ at $72^{\circ} \mathrm{C}$. The reaction products were then cooled to $50^{\circ} \mathrm{C}$ and subjected to a post-PCR melting cycle by increasing the temperature by $0.2^{\circ} \mathrm{C}$ every $10 \mathrm{~s}$, up to $95^{\circ} \mathrm{C}$. The comparative $\mathrm{C}_{\mathrm{T}}$ method was used to analyze the relative transcription levels of HolSMP after infection.

\section{Membrane protein extraction from SMP-infected host cells}

To identify the expression of HolSMP in SMP-infected $S$. suis, exponentially growing SS2-H cells were infected with phage SMP as described above. Samples, containing $10^{11}$ host cells, were collected as described above at 20, 40, 60, 80 and $100 \mathrm{~min}$ after infection. Cell pellets were frozen in liquid nitrogen immediately until required for extraction of membrane proteins. To prepare membrane fractions, harvested cells were suspended in $5 \mathrm{ml}$ ice-cold lysis buffer ( $300 \mathrm{mM} \mathrm{NaCl}, 50 \mathrm{mM}$ sodium phosphate, pH8.0), and sonicated on ice at $200 \mathrm{~W}$ for 50 cycles of 3 $\mathrm{s}$ on and $20 \mathrm{~s}$ off. The cell fragments were collected by centrifugation at $13,000 \times g$ for $1 \mathrm{~min}$. This process was repeated until cells were completely lysed. The collected supernatant was ultracentrifuged at $100,000 \times g$ for $1 \mathrm{~h}$ at $4^{\circ} \mathrm{C}$ to pellet membrane fragments. Each pellet was solubilized with $5 \mathrm{ml} \mathrm{ME}$ buffer (1\%Triton X-100, 10\% glycerine, $0.5 \mathrm{M} \mathrm{NaCl}, 35 \mathrm{mM} \mathrm{MgCl}$, $220 \mathrm{mM}$ Tris- $\mathrm{HCl}$, $\mathrm{pH}$ 8.0) and incubated for $12 \mathrm{~h}$ on ice with shaking [29]. The insoluble fraction was discarded after ultracentrifugation at $100,000 \times g$ for $1 \mathrm{~h}$ at $4^{\circ} \mathrm{C}$. Note that addition of lysozyme must be avoided.

\section{Protein expression and viability assays}

BL21(DE3)pLysS harboring plasmid pEXH1, designated BL21(DE3)pLysS (pEXH1), was inoculated and cultured to an optical density at $600 \mathrm{~nm}\left(\mathrm{OD}_{600}\right)$ of $0.5 \sim 0.6$. Protein expression was induced by addition of IPTG to a final concentration of $1 \mathrm{mM}$ and shaking at $30^{\circ} \mathrm{C}$ at $150 \mathrm{rpm}$. The growth of clones after induction was monitored by measuring $\mathrm{OD}_{600}$. For protein expression analysis, cells in $1 \mathrm{ml}$ cultures were suspended with 100 $\mu \mathrm{l} 1 \times$ tricine sample buffer $(1 \times \mathrm{TSB}, 50 \mathrm{mM}$ Tris- $\mathrm{HCl}$ [pH 6.8] containing $12 \%$ [w/v] glycerol, $4 \%$ [w/v] SDS, $2.5 \%[\mathrm{v} / \mathrm{v}]$ mercaptoethanol and $0.01 \%[\mathrm{w} / \mathrm{v}]$ bromophenol blue) and boiled for about $5 \mathrm{~min}$ to prepare total cellular protein samples. For viability assays, $20 \mu \mathrm{l}$ cultures of BL21(DE3)pLysS carrying plasmid pEXH1 was placed on ice at different time points after addition of IPTG. Each sample was serially diluted on ice and 100 $\mu \mathrm{l}$ dilutions were plated in triplicate on LB-Ap. Colonies from three separate experiments were counted after 12 to $16 \mathrm{~h}$ of incubation at $37^{\circ} \mathrm{C}$.

\section{Subcellular fractionation}

One litre culture of BL21(DE3)pLysS (pEXH1) induced for 40 min by IPTG was harvested by centrifuging at $13,000 \times g$ for $3 \mathrm{~min}$ at $4^{\circ} \mathrm{C}$. To prepare the cytoplasmic fraction, harvested cells were suspended in $5 \mathrm{ml}$ ice cold lysis buffer, sonicated on ice at $400 \mathrm{~W}$ for $20 \mathrm{~min}$, (3 s on/20 s off cycles), and ultracentrifuged at 100,000 $\times g$ for $1 \mathrm{~h}$ at $4^{\circ} \mathrm{C}$ to remove the membrane fraction. Membrane protein preparation from membrane pellets was performed with $5 \mathrm{ml} \mathrm{ME}$ buffer as described in membrane protein extraction from SMP-infected host cells. Both cytoplasmic fraction and membrane fraction samples were mixed with $2 \times$ tricine sample buffer and boiled.

\section{Tricine-SDS-PAGE and western blots}

HolSMP was separated by tricine-SDS-PAGE and examined by western blotting. For tricine-SDS-PAGE, the protein samples were resolved on $20 \%(\mathrm{w} / \mathrm{v})$ polyacrylamide gels as previously described [30]. The gel was stained with Coomassie blue or directly used to transfer proteins onto a nitrocellulose membrane by electroblotting. The antibody against a recombinant protein corresponding to $\mathrm{TMD}_{2}-\mathrm{TMD}_{3}-\mathrm{C}$-terminal sequence of HolSMP was raised in mouse in our lab previously. For immunodetection of HolSMP, the antibody against HolSMP (1:1000 dilution) and the goat anti-mouse immunoglobulin conjugated to horseradish peroxidase (1:2500 dilution; Immunology Consultants Laboratory, Inc.) were used as the primary and secondary antibodies, respectively. The western blots were analyzed with DAB colorimetric western blot kit (Rockland).

\section{Transmission electron microscopy}

Culture samples were collected every $5 \mathrm{~min}$ in the first hour after the addition of IPTG and centrifuged at 1,160 $\times g$ for $3 \mathrm{~min}$ to pellet cells. The pellets were re-suspended in $2.5 \%$ glutaraldehyde in $0.1 \mathrm{M}$ PBS (pH 7.4). Cells were fixed at $4^{\circ} \mathrm{C}$ for $30 \mathrm{~min}$ and centrifuged at $1,160 \times g$ for $1 \mathrm{~min}$. Thin sections of the cells were processed and examined at 60,000 $\times$ magnification with a Hitachi H-600 transmission electron microscope.

\section{Co-expression of HolSMP and LySMP in E. coli}

To explain the physiological role of HolSMP, HolSMP was co-expressed with LySMP. The HindIII-SphI fragment containing LySMP from pEXL and a negative control sequence from $\mathrm{pEX}$ were inserted into plasmid pACYC184. The resulting plasmids were designated pACEXL and pACEX. The chloramphenicol-resistant plasmid pACYC184 harboring the p15A origin of replication was compatible with colE1 of vector pET-32a $(+)$ [31]. Therefore, the recombinant plasmids pACEXL and pACEX were compatible with pEXH1. The E. coli BL21 
(DE3) strains harboring plasmid combinations pEXH1 +pACEXL (harboring both HolSMP and LySMP), pEXH1+pACEX (harboring HolSMP only) and pACEXL $+\mathrm{pEX}$ (harboring LySMP only) were grown overnight in LB-Ap-Cm. The strains were diluted (1:100) with fresh medium and cultured to an $\mathrm{OD}_{600}$ of 0.6. Expression of the genes was induced by addition of IPTG, and the growth of clones was monitored by measuring $\mathrm{OD}_{600}$.

\section{Complementation of $\lambda$ Sam 7 lysis function}

BL21(DE3)pLysS (pEXH1) was inoculated and cultured to an $\mathrm{OD}_{600}$ of about 0.5 in LB-Ap. A $200-\mu \mathrm{l}$ BL21 (DE3)pLysS culture was infected at $37^{\circ} \mathrm{C}$ for $15 \mathrm{~min}$ with $10 \mu \mathrm{l}$ bacteriophage $\lambda$ cI857 $\operatorname{Sam} 7\left(10^{5}\right.$ plaque forming units $/ \mathrm{ml}$ ). The E. coli BL21(DE3)pLysS and phage were mixed with $5 \mathrm{ml}$ of soft agar containing $0.1 \mathrm{mM}$ IPTG and $100 \mu \mathrm{g} / \mathrm{ml}$ ampicillin, and quickly poured onto LB-Ap plates. Addition of $0.1 \mathrm{mM}$ IPTG to the soft agar induced expression of HolSMP at sublethal levels for BL21(DE3)pLysS harboring plasmid pEXH1 unless the R lysin of $\lambda$ cI857 Sam7 was also present. The plates were incubated face up at $37^{\circ} \mathrm{C}$ to encourage the formation of plaques, and the number of plaques was determined after overnight incubation. BL21(DE3)pLysS with plasmid pET-32a(+) and VCS257 were used as controls. Before infection, freshly cultured VCS257 was gently resuspended and diluted to an $\mathrm{OD}_{600}$ of 0.5 with sterile $10 \mathrm{mM} \mathrm{MgSO}_{4}$ after centrifugation at $500 \times g$ for $10 \mathrm{~min}$. Antibiotic was not added to the soft agar or plates to culture VCS257.

\section{Abbreviations \\ E. coli: Escherichia coli; GAPDH: Glyceraldehydes-3-phosphate dehydrogenase gene; KCN: Potassium cyanide; IPTG: $\beta$-D-thiogalactopyranoside; LB: Luria- Bertani medium; LB-Ap: LB supplemented with ampicillin $(100 \mathrm{\mu g} / \mathrm{ml}) ; \mathrm{LB}-$ Ap-Cm: LB supplemented with ampicillin $(100 \mu \mathrm{g} / \mathrm{ml})$ and chloramphenicol $(30 \mu \mathrm{g} / \mathrm{ml})$; LB-Cm: LB supplemented with chloramphenicol $(30 \mathrm{\mu g} / \mathrm{ml}) ;$ ORF Open reading frame; RBS: Ribosome-binding site; sdi: Site-directed initiation S. suis: Streptococcus suis; THB: Todd-Hewitt broth; TMD: Transmembrane domain.}

\section{Acknowledgements}

The authors would like to thank Prof. Lu Chengping from Nanjing Agricultural University for providing constructive suggestions. The research was supported by National Natural Science Foundation (31172381) and fund from State Key Laboratory of Veterinary Etiological Biology (SKLVEB2010KFKT004).

\section{Authors' contributions}

YBS and JHS carried out the design of the study, analyzed the data and drafted the manuscript. WHJ performed the plaque and viability assays. DB participated in the transmission electron microscopy analysis. XPM conceived of the study, participated in its design and coordination and helped to draft the manuscript. YXY and HAW participated in the design of the study. All authors read and approved the final manuscript.

\section{Competing interests}

The authors declare that they have no competing interests.
Received: 16 October 2011 Accepted: 22 March 2012

Published: 22 March 2012

\section{References}

1. Gründling A, Bläsi U, Young R: Biochemical and genetic evidence for three transmembrane domains in the class I holin, lambda S. J Biol Chem 2000, 275:769-776

2. Takáč M, Witte A, Bläsi U: Functional analysis of the lysis genes of Staphylococcus aureu phage P68 in Escherichia coli. Microbiology 2005 151:2331-2342.

3. Pang T, Savva CG, Fleming KG, Struck DK, Young R: Structure of the lethal phage pinhole. Proc Natl Acad Sci USA 2009, 106:18966-18971.

4. Zimmer M, Vukov N, Scherer S, Loessner MJ: The murein hydrolase of the bacteriophage phi3626 dual lysis system is active against all tested Clostridium perfringen strains. Appl Environ Microbiol 2002, 68:5311-5317.

5. Seal BS, Fouts DE, Simmons M, Garrish JK, Kuntz RL, Woolsey R, Schegg KM, Kropinski AM, Ackermann HW, Siragusa GR: Clostridium perfringen bacteriophages PhiCP390 and PhiCP26F: genomic organization and proteomic analysis of the virions. Arch Virol 2011, 156:25-35.

6. Tran TA, Struck DK, Young R: Periplasmic domains define holin-antiholin interactions in t4 lysis inhibition. J Bacteriol 2005, 187:6631-6640.

7. White $R$, Tran TA, Dankenbring CA, Deaton J, Young R: The N-terminal transmembrane domain of lambda $S$ is required for holin but not antiholin function. J Bacteriol 2010, 192:725-733.

8. Krupovič M, Bamford DH: Holin of bacteriophage lambda: structural insights into a membrane lesion. Mol Microbiol 2008, 69:781-783.

9. Golec P, Wiczk A, Majchrzyk A, Łoś JM, Węgrzyn G, Łoś M: A role for accessory genes rl.-1 and $\mathrm{rl} .1$ in the regulation of lysis inhibition by bacteriophage T4. Virus Genes 2010, 41:459-468.

10. Rydman PS, Bamford DH: Identification and mutational analysis of bacteriophage PRD1 holin protein P35. J Bacteriol 2003, 185:3795-3803.

11. Žiedaite G, Daugelavičius R, Bamford JK, Bamford DH: The Holin protein of bacteriophage PRD1 forms a pore for small-molecule and endolysin translocation. J Bacteriol 2005, 187:5397-5405.

12. Horii T, Suzuki Y, Kobayashi M: Characterization of a holin (HolNU3-1) in methicillin-resistant Staphylococcus aureu host. FEMS Immunol Med Microbiol 2002, 34:307-310.

13. Kashige N, Nakashima Y, Miake F, Watanabe K: Cloning, sequence analysis, and expression of Lactobacillus case phage PL-1 lysis genes. Arch Virol $2000,145 \cdot 1521-1534$

14. Labrie S, Vukov N, Loessner MJ, Moineau S: Distribution and composition of the lysis cassette of Lactococcus lacti phages and functional analysis of bacteriophage ul36 holin. FEMS Microbiol Lett 2004, 233:37-43.

15. Wang $S$, Kong J, Zhang $X$ : Identification and characterization of the twocomponent cell lysis cassette encoded by temperate bacteriophage phiPYB5 of Lactobacillus fermentu. J Appl Microbiol 2008, 105:1939-1944.

16. Sheehan MM, Stanley E, Fitzgerald GF, van Sinderen D: Identification and characterization of a lysis module present in a large proportion of bacteriophages infecting Streptococcus thermophilu. Appl Environ Microbiol 1999, 65:569-577.

17. Diaz E, Munthali M, Lunsdorf $H$, Holtje JV, Timmis KN: The two-step lysis system of pneumococcal bacteriophage EJ-1 is functional in gramnegativebacteria: triggering of the major pneumococcal autolysin in Escherichia col. Mol Microbiol 1996, 19:667-681.

18. Martín AC, López R, García P: Functional analysis of the two-gene lysis system of the pneumococcal phage $\mathrm{Cp}-1$ in homologous and heterologous host cells. J Bacteriol 1998, 180:210-217.

19. Vukov N, Moll I, Bläsi U, Scherer S, Loessner MJ: Functional regulation of the Listeria monocytogene bacteriophage A118 holin by an intragenic inhibitor lacking the first transmembrane domain. Mol Microbiol 2003, 48:173-186.

20. Loessner MJ, Maier SK, Daubek-Puza H, Wendlinger G, Scherer S: Three Bacillus cereu bacteriophage endolysins are unrelated but reveal high homology to cell wall hydrolases from different bacilli. J Bacteriol 1997, 179:2845-2851.

21. Gottschalk M, Segura M: The pathogenesis of the meningitis caused by Streptococcus suis: the unresolved questions. Vet Microbio/ 2000, 76:259-272.

22. Ma YL, Lu CP: Isolation and identification of a bacteriophage capable of infecting Streptococcus sui type 2 strains. Vet Microbiol 2008, 132:340-347. 
23. Fenton M, Ross P, McAuliffe O, O'Mahony J, Coffey A: Recombinant bacteriophage lysins as antibacterials. Bioeng Bugs 2010, 1:6-16.

24. Wang Y, Sun JH, Lu CP: Purified recombinant phage lysin LySMP: an extensive spectrum of lytic activity for swine streptococci. Curr Microbiol 2009, 58:609-615.

25. Meng X, Shi Y, Ji W, Meng X, Zhang J, Wang H, Lu C, Sun J, Yan Y: Application of a bacteriophage lysin to disrupt biofilms formed by the animal pathogen, Streptococcus suis. Appl Environ Microbiol 2011, 77:8272-8279.

26. Steiner M, Bläsi U: Charged amino-terminal amino acids affect the lethal capacity of Lambda lysis proteins S107 and S105. Mol Microbiol 1993, 8:525-533.

27. Gründling A, Smith DL, Bläsi U, Young R: Dimerization between the holin and holin inhibitor of phage $\lambda$. J Bacteriol 2000, 182:6075-6081.

28. Sambrook J, Russell DW: Bacteriophage $\lambda$ and its vectors: purification of bacteriophage $\lambda$ particles by centrifugation through a glycerol step gradient. In Molecular Cloning: A Laboratory Manual. Volume 1.. 3 edition. Edited by: Jan Argentine. New York: Cold Spring Harbor Laboratory Press: 2001:52-53.

29. Smith DL, Chang CY, Young R: The lambda holin accumulates beyond thelethal triggering concentration under hyperexpression conditions. Gene Expr 1998, 7:39-52.

30. Chang CY, Nam K, Young R: gene expression and the timing of lysis by bacteriophage lambda. J Bacteriol 1995, 177:3283-3294.

31. Farkašovská J, Godány A, Vlček Č: Identification of a holin encoded by the Streptomyces aureofacien phage micro1/6; functional analysis in Escherichia col system. Folia Microbiol (Praha) 2004, 49:679-684.

doi:10.1186/1743-422X-9-70

Cite this article as: Shi et al: Characterization and determination of holin protein of Streptococcus suis bacteriophage SMP in heterologous host. Virology Journal 2012 9:70.

\section{Submit your next manuscript to BioMed Central} and take full advantage of:

- Convenient online submission

- Thorough peer review

- No space constraints or color figure charges

- Immediate publication on acceptance

- Inclusion in PubMed, CAS, Scopus and Google Scholar

- Research which is freely available for redistribution

Submit your manuscript at www.biomedcentral.com/submit
Biomed Central 